\title{
Molecular Oxygen Formation in Interstellar Ices Does Not Require Tunneling
}

\author{
Marco Pezzella, Oliver T. Unke, and Markus Meuwly* \\ Department of Chemistry, University of Basel, \\ Klingelbergstrasse 80, 4056 Basel, Switzerland \\ E-mail: m.meuwly@unibas.ch
}

\begin{abstract}
Formation of molecular oxygen in and on amorphous ice in the interstellar medium requires oxygen diffusion to take place. Recent experiments suggest that this process involves quantum tunneling of the oxygen atoms at sufficiently low temperatures. Fitting experimental diffusion rates between 6 and $25 \mathrm{~K}$ to an expression that accounts for the roughness of the surface yields excellent agreement. The molecular dynamics of adsorbed oxygen is characterized by rapid intrasite dynamics followed by intersite transitions over distances of $\sim 10 \AA$. Explicit simulations using a realistic free energy surface for oxygen diffusion on amorphous ice down to $10 \mathrm{~K}$ show that quantum tunneling is not required for mobility of adsorbed oxygen. This is confirmed by comparing quantum and classical simulations using the same free energy surface. The ratio of diffusional and desorption energy $E_{\text {dif }} / E_{\text {des }}=275 / 1082 \approx 0.3$ is at the lower end of typically used values but still consistent with assumptions made in models for interstellar chemistry.
\end{abstract}




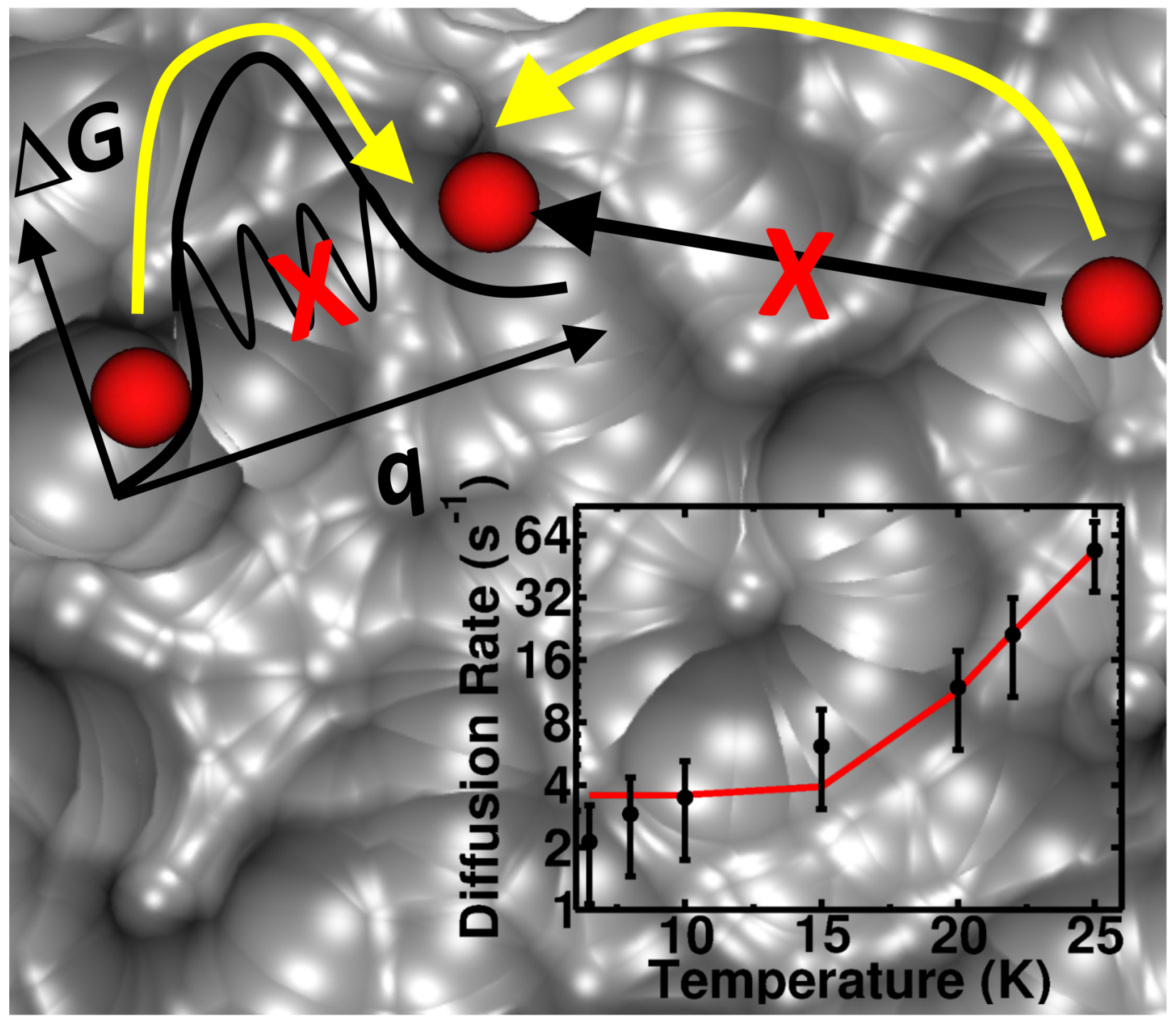

Figure 1: TOC figure 
The diffusion of oxygen atoms in and on interstellar ice grains is of fundamental importance for the formation of $\mathrm{CO}_{2}$ and molecular oxygen $\mathrm{O}_{2} \cdot{ }^{1-3}$ In addition, O-diffusion has also been implicated in formation of ozone in laboratory experiments ${ }^{4,5}$ within a LangmuirHinshelwood mechanism (surface diffusion) and has been proposed to be relevant for interstellar grains ${ }^{1}$ eventually leading to the formation of water ${ }^{5}$ although the major waterformation pathway involves mobile hydrogen atoms.

In all these cases the diffusion of atomic oxygen is a primary driver for forming di- or triatomic molecules under conditions typical for interstellar environments. These include higher temperatures $(T \approx 50 \mathrm{~K})$ for translucent and diffuse clouds and temperatures $T \approx 10 \mathrm{~K}$ for dense molecular clouds. ${ }^{1,6}$ Questions of particular relevance pertain to the diffusional barrier for intersite migration of oxygen atoms on amorphous solid water (ASW) and their desorption energies away from the surface. For this, experiments based on temperature programmed desorption have been used. ${ }^{4,7}$

For analyzing the diffusion rate (i.e. the probability for one hop between different adsorption sites in $\mathrm{s}^{-1}$, which is referred to as $D$ in Ref. ${ }^{4}$ ) of atomic oxygen on ASW, either an Arrhenius expression $\left(D(T) \sim A e^{\left(-\beta E_{\text {dif }}\right)}\right.$ where $E_{\text {dif }}$ is a conformationally averaged diffusional barrier and $\left.\beta=1 /\left(k_{B} T\right)\right)$ or an expression accounting for tunneling through a square well barrier was used. ${ }^{4,7}$ A diffusion coefficient $D_{\text {dif }}$ (in units of length ${ }^{2} /$ time) can be converted into a diffusion rate $D$ (in $1 /$ time) through $D=D_{\text {dif }} / a^{2}$ where $a^{2}$ is a typical area sampled by the adsorbate between consecutive hops (see Figure S1). Typically, $a^{2}$ is of the order of $10 \AA^{2}$ which corresponds to $10^{15}$ adsorption sites $/ \mathrm{cm}^{2}{ }^{8}$ Using temperature-programmed desorption, the formation of molecular oxygen and ozone from atomic oxygen was studied on amorphous solid water and crystalline water. ${ }^{4}$ From monitoring the relative amounts of the two molecular species $n\left(\mathrm{O}_{3}\right) / n\left(\mathrm{O}_{2}\right)$ as a function of temperature (between 6 and $25 \mathrm{~K}$ ), the oxygen diffusion rate as a function of temperature was obtained. This data was analyzed 
by assuming an Arrhenius law with an activation energy of $450 \mathrm{~K}$ and a prefactor of $10^{12}$ $\mathrm{s}^{-1}$ which was unable to describe the temperature dependence (see green line in Figure 2). ${ }^{4,7}$ Alternatively, a model that accounts for tunneling through a square barrier was used which, however, did also not yield a satisfactory fit using a typical activation energy of $500 \mathrm{~K}$ and a barrier width of $1 \AA .{ }^{4}$

However, it is to be noted that such models assume homogeneous, non-corrugated (i.e. "smooth") surfaces on which the diffusing particle moves. Once the surface contains variations on atomic length scales ("roughness" of scale $\epsilon$, see Figure 2) as is typical for ASW, the temperature dependence of the diffusion rate may change. Diffusion in a rough, onedimensional potential was investigated by Zwanzig who found that the motion of a particle on a rough potential energy surface (PES) is characterized by an effective diffusion coefficient $D_{\text {dif }}^{*} \cdot{ }^{9}$ If the roughness of the surface is random, independent of position $x$ and Gaussian-distributed in $\epsilon$ (the scale of the roughness), then the effective diffusion coefficient is $D_{\text {dif }}^{*}=D_{\text {dif }} e^{-(\beta \epsilon)^{2}}$ where $D_{\text {dif }}$ is the diffusion coefficient on the surface with zero roughness. On the other hand, for a periodic perturbation $\propto \epsilon \cos (q x)$ of the roughness with periodicity $q$ the effective diffusion coefficient at low temperatures is $D_{\text {dif }}^{*}=D_{\text {dif }} e^{-(2 \beta \epsilon)}$, i.e. an Arrhenius dependence.

Because an Arrhenius expression does manifestly not describe the diffusion at low temperatures correctly, we used an expression $D^{*}=D_{0}+D e^{-(\beta \epsilon)^{2}}$ in which $D_{0}, D$ and $\epsilon$ are adjustable parameters to fit the experimental data from Ref. ${ }^{4}$ Figure 2 demonstrates that such an expression faithfully describes the experiments down to $6 \mathrm{~K}$ whereby the parameter values are $D_{0}=3.75 \mathrm{~s}^{-1}, D=8.83 \times 10^{4} \mathrm{~s}^{-1}$, and $\epsilon=93.4 \mathrm{~K}$. For comparison, fits with $D_{0}=0$ and the Arrhenius expression used in Ref. ${ }^{4}$ are also shown. Diffusivities larger than 0 at very low temperatures $(\leq 1 \mathrm{~K})$ have, for example, been observed for atom diffusion in body centered tungsten. ${ }^{10,11}$ Finite diffusivities at such low temperatures $(<1 \mathrm{~K})$ have been 
primarily associated with zero point motion. ${ }^{11}$

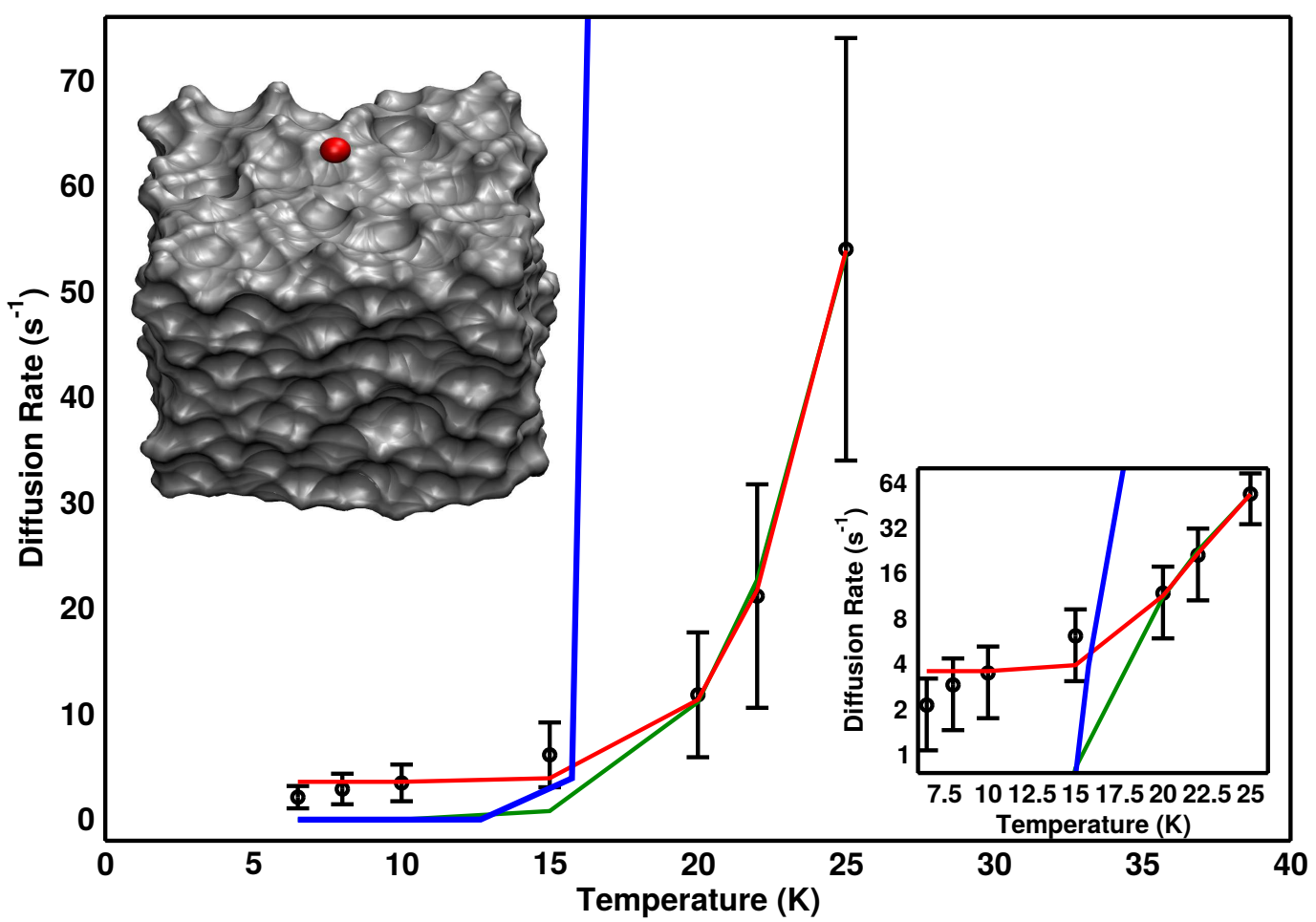

Figure 2: Different models for oxygen diffusion at low temperatures (6-25 K). Experimental points and error bars are those from Ref. ${ }^{4}$ The blue curve is the Arrhenius expression using the parameters from Ref. ${ }^{4}$ (Figure $3, D=A e^{(-\beta \epsilon)}$ with $A=10^{12} \mathrm{~s}^{-1}$ and $\epsilon=450 \mathrm{~K}$ ) which fails to describe the experimental data. Fitting to an expression suggested in Ref. ${ }^{9}$ $\left(D^{*}=D e^{-(\epsilon / T)^{2}}, D=2.88 \times 10^{4} \pm 2.61 \times 10^{4} \mathrm{~s}^{-1}, \epsilon=78.6 \pm 11.0 \mathrm{~K}\right.$, green line) describes the data down to $20 \mathrm{~K}$. Allowing for finite diffusion down to very low temperatures $\left(D^{*}=\right.$ $D_{0}+D e^{-(\epsilon / T)^{2}}, D_{0}=3.75 \pm 0.70 \mathrm{~s}^{-1}, D=8.83 \times 10^{4} \pm 4.82 \times 10^{4} \mathrm{~s}^{-1}, \epsilon=93.4 \pm 6.8$ $\mathrm{K}$, red curve) reproduces the experimental data to $6 \mathrm{~K}$. The inset reports the $y$-axis on a logarithmic scale.

To further corroborate the finding that no major corrections due to tunneling are required, atomistic simulations together with quantum dynamical calculations for the translational motion of the oxygen atom were carried out. Molecular Dynamics (MD) and Monte Carlo (MC) simulations were performed using CHARMM. ${ }^{12}$ The initial ASW structure was generated as described previously. ${ }^{13}$ Starting from a TIP3P ${ }^{14}$ water box $\left(31 \times 31 \times 50 \AA^{3}\right)$, 
equilibrated at $300 \mathrm{~K}$, the system is first quenched to $50 \mathrm{~K}$, then equilibrated in the $N p T$ ensemble, followed by further equilibration with $N V T$ using periodic boundary conditions. A cutoff of $13 \AA$ is applied to the non-bonded interactions. All production MD simulations are performed in the microcanonical $(N V E)$ ensemble with a time step of 1 fs. For both simulation temperatures of 10 and $50 \mathrm{~K}$, respectively, heating and equilibration runs were performed for 10 and $50 \mathrm{ps}$, respectively. At $10 \mathrm{~K}$ only a single production simulation is run for $900 \mathrm{~ns}$, due the low mobility of the oxygen atom on the surface. Increasing the temperature to $50 \mathrm{~K}$ leads to much wider sampling and 10 independent simulations, each 900 ns long, were run. Bonds involving hydrogen atoms were constrained using SHAKE. ${ }^{15}$

For determining the nonbonded parameters for oxygen, 34 clusters (from an equilibrium MD simulation at $50 \mathrm{~K}$ ) consisting of 12 water molecules interacting with one oxygen atom were extracted and total energies at the MP2/aug-cc-pVDZ level of theory using Gaussian09 ${ }^{16}$ were determined. Next, the oxygen atom van der Waals parameters $r_{\min , \mathrm{O}}$ and $\epsilon_{\mathrm{O}}$ were fitted using a simplex algorithm to best reproduce the target energies which yielded $r_{\min , \mathrm{O}}=1.62$ $\AA$ and $\epsilon_{\mathrm{O}}=-0.518 \mathrm{kcal} / \mathrm{mol}$. This parametrization gives oxygen desorption energies of $2.15 \mathrm{kcal} / \mathrm{mol}(1082 \mathrm{~K})$ in good agreement with values of $1380 \mathrm{~K}$ derived from temperature programmed desorption experiments. ${ }^{7}$

Using this parametrization, the 2-dimensional free energy surface $G(x, y)$ was determined from Monte Carlo $(\mathrm{MC})$ simulations at $50 \mathrm{~K}$ on an equally spaced grid $\left(31 \times 31 \AA^{2}\right)$. For each of the 961 grid points, $10^{8} \mathrm{MC}$ steps were run to determine the probability distribution $P(x, y)$ from which $G=-R T \ln (P)$ was determined. The interaction of the oxygen atom with the water surface is sufficiently strong to preclude exhaustive sampling of the entire $G(x, y)$ (only $60 \%$ of the grid is sampled). Therefore, the same MC simulations were repeated with the original ("orig") CHARMM36 parametrization for the oxygen atom. For this force field the oxygen desorption energy is reduced to $\sim 1 \mathrm{kcal} / \mathrm{mol}$ which allows complete and 
exhaustive sampling of $G_{\text {orig }}(x, y)$ due to the smaller desorption energy which also lowers the intersite barrier. The two free energy surfaces can be readily related for the grid points that are sufficiently sampled (60\% of all grid points) with a correlation coefficient of 0.925 and hence the less sampled regions using the correct, present parametrization for the oxygen atom can be extrapolated from $G_{\text {orig }}(x, y)$, as shown in Figure $\mathrm{S} 2$.

In addition, explicit MD simulations were carried out at $10 \mathrm{~K}$ and $50 \mathrm{~K}$ using the refined nonbonded parameters for the oxygen atom. For $10 \mathrm{~K}$ the oxygen atom diffuses between neighboring minima during $900 \mathrm{~ns}$, see Figure 3. Increasing the temperature to $50 \mathrm{~K}$ the oxygen atom samples an extended path (length $62.4 \AA$ (minima 1 to 8 and 17 to 26 , see Figure 3A)) within 900 ns of explicit MD simulations. Hence, in translucent and diffuse clouds oxygen diffusion is expected to occur. The average value of the free energy profile is indicated by the dashed red line whereas the average barrier height $(0.55 \mathrm{kcal} / \mathrm{mol}$ equivalent to $275 \mathrm{~K}$ ) is the dashed green line above the average energy. As can be seen, many barriers along the path are below the average barrier height. At $50 \mathrm{~K}$ the average dwell time in a local minimum is $\sim 5 \mathrm{~ns}$ which suggests facile diffusion at such temperatures on comparatively short time scales.

In order to explicitly address the question whether or not quantum effects are relevant for oxygen diffusion, the one dimensional Schrödinger equation for the translational motion of the oxygen atom was solved for part of the free energy profile from Figure 3 (minima 23, 24, 25, see Figure 4). For comparison, classical MD simulations solving Newton's equations of motion were also carried out using the same free energy profile. Parabolic walls were added at the outer boundaries in order to confine the motion of the oxygen atom.

For following the quantum mechanical time evolution of the system, the time-dependent 


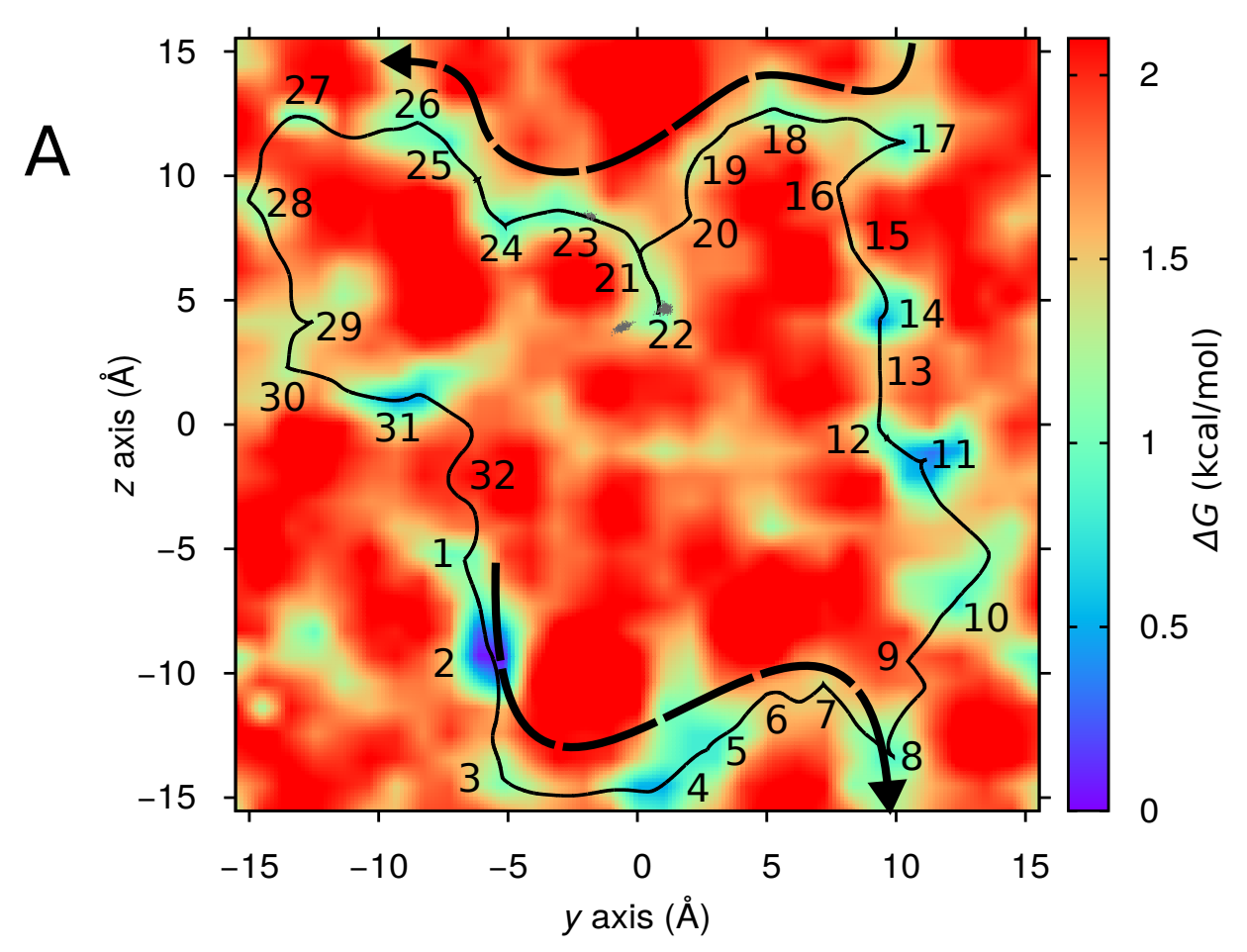

B

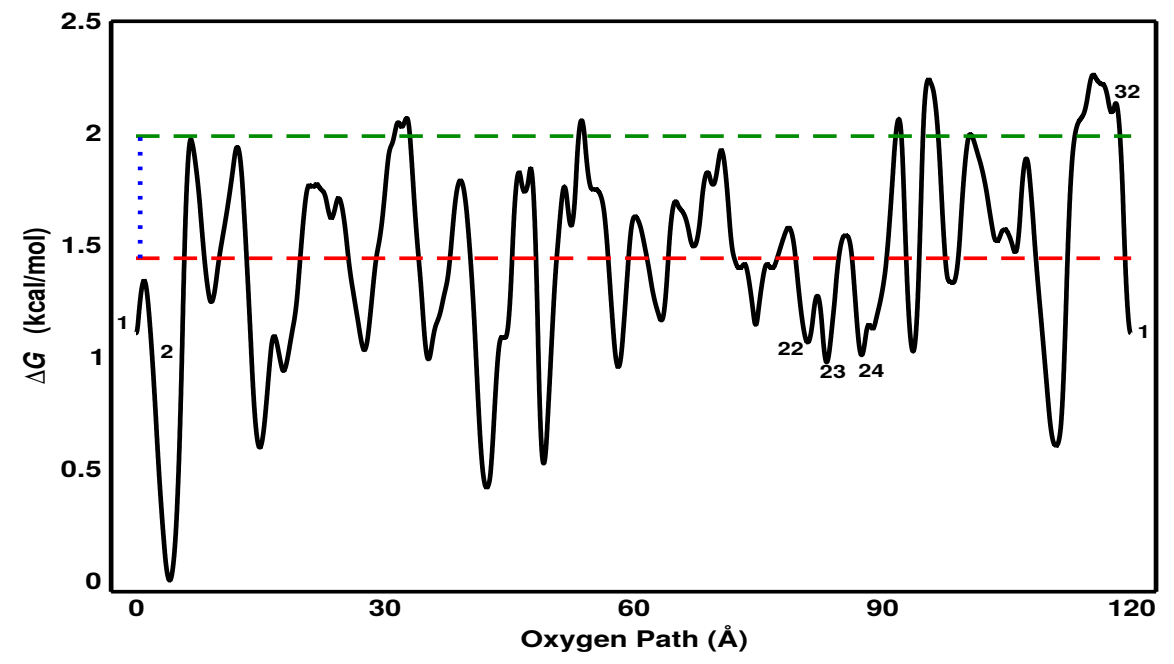

Figure 3: A typical oxygen diffusion path. Panel A shows the 2-dimensional free energy surface $G(x, y)$. All the minima of the free energy surface are labeled from 1 to 32 . They form a continuous closed loop of length $120 \AA$. The typical region visited during one single $500 \mathrm{~ns} \mathrm{MD}$ simulation at $50 \mathrm{~K}$ is indicated by a dashed, arrowed line and includes minima 1 to 8 and (due to periodic boundaries) 17 to 26. Even for a $900 \mathrm{~ns}$ simulation at $10 \mathrm{~K}$ (grey dots) several minima (22 to 23) are sampled. Panel B is the 1-dimensional projection of the closed loop from panel $\mathrm{A}$ with the path starting and ending at minimum number 1 . The average free energy is indicated by the red dashed line and the average barrier height (green dashed line $)$ is $275 \mathrm{~K}(0.55 \mathrm{kcal} / \mathrm{mol})$. 
Schrödinger equation:

$$
i \hbar \frac{\partial}{\partial t} \Psi(x, t)=\hat{H} \Psi(x, t)
$$

was solved using the Crank Nicholson (CN) method. ${ }^{17}$ Here, $x$ is the position, $t$ is time, $\hat{H}$ the Hamiltonian and $\Psi(x, t)$ the wave function. The CN method is a second order, symplectic method which conserves total energy and provides high accuracy. Simulations used a time step with $\delta t=0.2 \mathrm{fs}$ and a grid spacing of $\delta x=0.01 \mathrm{a}_{0}$. The original width of the ground state wave function was 2.048 a.u. which is the width of the harmonic approximation to the potential around the minimum.

For the classical molecular dynamics simulations the initial position of all particles was $\delta\left(x-x_{0}\right)$, where $x_{0}=2.279 \AA$ is the minimum of the 1-dimensional energy profile in region 1 (see Figure 4), and velocities were sampled from a Maxwell-Boltzmann distribution at 50 $\mathrm{K}$. The classical equations for motion were propagated with a $1 \mathrm{fs}$ time step using a Velocity Verlet algorithm. ${ }^{18}$ Each dynamics was run for 5 ns and averaged over 10000 independent simulations. For solving the 1-dimensional time-dependent Schrödinger equation using the CN method 1000 simulations were initialized according to a Maxwell Boltzmann distribution at $50 \mathrm{~K}$. For each time frame the amplitudes were Boltzmann-averaged to obtain the total probability distribution.

Both, classical (panel C) and quantum simulations (panel D) reach a steady distribution after 10 ps (classical) and 20 ps (quantum), respectively, showing a slightly larger probability $\left(10^{-3}\right.$ vs. $5 \times 10^{-4}$ for region 3 and $10^{-2}$ vs. $3 \times 10^{-2}$ for region 2$)$ if quantum effects are included. At the end ( $5 \mathrm{~ns}$ ) of both simulations the probability remains highly localized in region 1 with just a small portion able to overcome the barriers. This reflects the fact that the system is unable to reach thermal equilibrium at $50 \mathrm{~K}$ for both the potential and the free energy because no energy exchange with the environment can take place and only particles with sufficient initial velocity eventually overcome the barrier between the global 


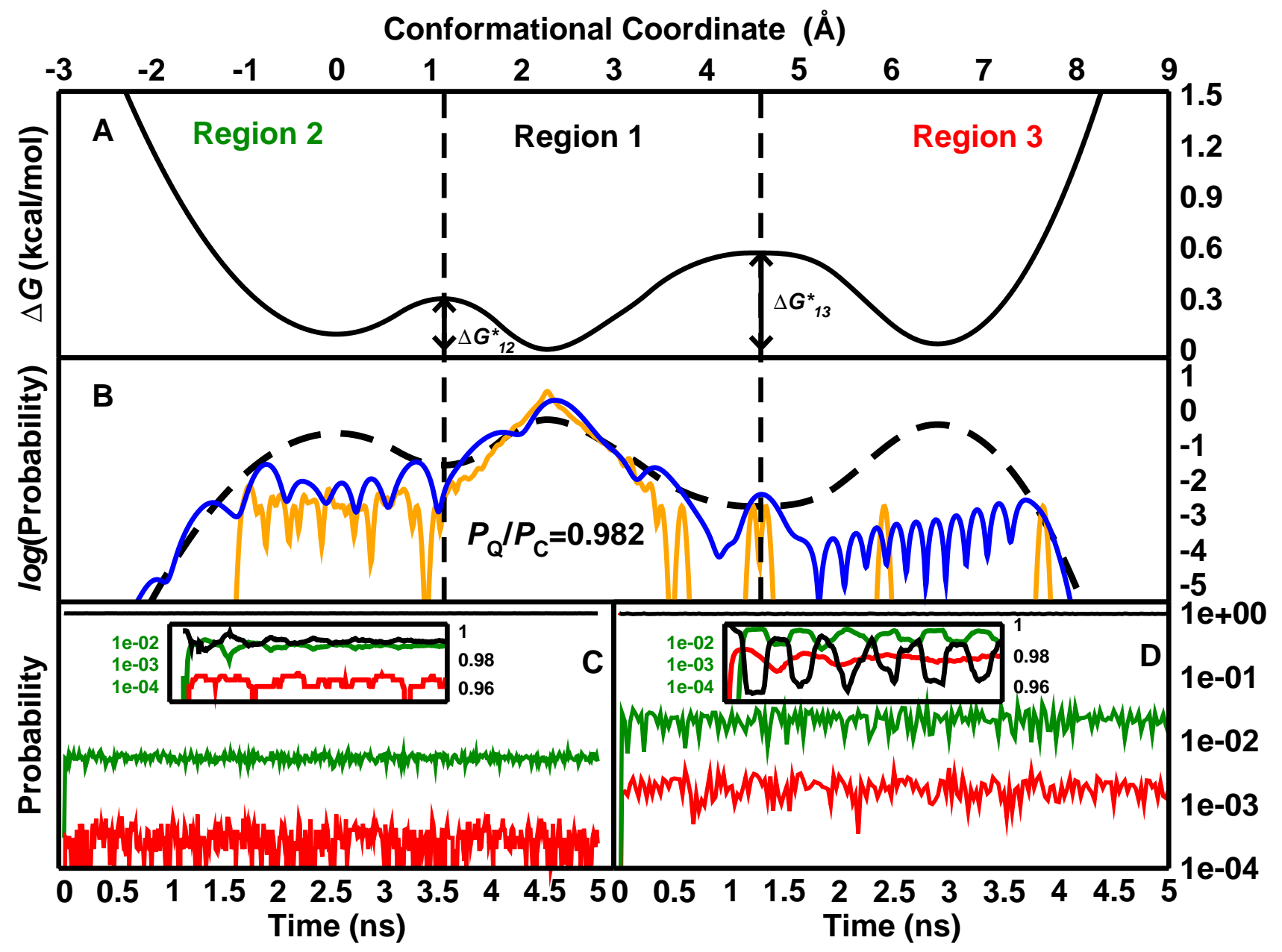

Figure 4: Panel A: Free energy curve for the path between minima 23 and 25 (regions 2 (green), 1 (black), and 3 (red), respectively) from the oxygen diffusion path in Figure 3. This particular path is chosen because it is representative for the average barrier height (275 $\mathrm{K}$ ) of the free energy surface. The barrier heights between regions $(1,2)$ and $(1,3)$ are $\Delta G_{12}=150 \mathrm{~K}$ and $\Delta G_{13}=256 \mathrm{~K}$, respectively. Panel B: Probability distribution after $5 \mathrm{~ns}$ of CN (blue) and classical MD simulations (orange) along with the equilibrium distribution (dashed black). The ratio of their amplitudes in region 1 is 0.98 indicating close agreement for quantum and classical dynamics on a time scale of $5 \mathrm{~ns}$. Using a $N V E$ ensemble the equilibrium probability is not reached. Panels $\mathrm{C}$ and $\mathrm{D}$ : probability distribution in regions 1 (black), 2 (green), and 3 (red) from panel A for classical MD (panel C, as number of particle per region) and $\mathrm{CN}$ (panel $\mathrm{D}$, as wave packet density per region). Amplitudes are reported on a logarithmic scale (for linear scale see Figure S3). Results in panel C are from classical $N V E$ MD simulations of 10000 oxygen atoms at $50 \mathrm{~K}$. Results in panel D are from a quantum mechanical simulation of a Boltzmann-averaged oxygen over 1000 simulations at $50 \mathrm{~K}$. The insets in the panels show the results from the first $10 \mathrm{ps}$ of simulation. The black scale refers to the black trace whereas the green scale corresponds to the green and red traces. 
minimum and the neighbouring local minima. Hence, the classical and quantum simulations are consistent with one another and suggest that tunneling plays a minor role. Remaining deviations from purely classical behaviour, e. g. a value of $D_{0} \neq 0$ in the expression for the diffusion rate $D^{*}=D_{0}+D e^{-(\epsilon / T)^{2}}$, are likely to be related to zero point motion.

Oxygen diffusion on a rough potential energy surface as provided by ASW allows for facile diffusion of the adsorbate down to temperatures relevant to interstellar chemistry. The parametrization for oxygen interacting with ASW used in this work correctly describes the average desorption energy of oxygen from water. Fitting the temperature dependence of the experimentally measured diffusion rates ${ }^{4}$ to an expression that accounts for roughness of the underlying PES yields a typical scale of the roughness of $\epsilon \sim 100 \mathrm{~K}$ which compares with typical average barrier heights of $E_{\text {dif }}=275 \mathrm{~K}$. Direct comparison of these two quantities should be done with care as they originate from two quite different analyses: $\epsilon$ is an effective roughness from a fit of experimental data sampling a large number of transitions and including the actual dynamics whereas $E_{\text {dif }}$ is an average barrier height experienced by the diffusing oxygen from a few ten transitions (see Figure 3B). Furthermore, the morphologies of the ASW surfaces from experiments and in the simulations are likely to differ. Nevertheless, both analyses clearly establish the existence of surface roughness and it is encouraging that the magnitude of the two effective parameters $\epsilon$ and $E_{\text {dif }}$, characterizing the surface corrugation are within less than a factor of three given the differences in the systems investigated. Explicit quantum and classical simulations for the translational motion of the oxygen atom on the free energy surface demonstrate that quantum and classical simulations on the 5 ns time scale agree well and tunneling does not play a role. This is particularly true when considering that rough surfaces tend to suppress tunneling effects.

The ratio of diffusional versus desorption barrier $E_{\text {dif }} / E_{\text {des }}=275 / 1082 \approx 0.3$ found in the present work (from sampling an exemplary circular path) is somewhat lower than that 
recently proposed from temperature desorption experiments $(0.55)^{7}$ but similar to other typically used ratios of 0.3 to $0.4{ }^{19}$ This ratio may depend on the surface morphology and change if longer simulations with more extensive sampling are carried out. Furthermore, the diffusion barrier height $E_{\text {dif }}=275 \mathrm{~K}$ is sufficiently low to yield comparatively rapid mobility at low temperatures. Such a barrier corresponds to $\approx 10^{4}$ hops per second (based on an Arrhenius-dependence with a frequency factor of $\left.10^{12} \mathrm{~s}^{-1}\right)^{7}$ and is consistent with values for $D \sim 10^{4}$ to $10^{5} \mathrm{~s}^{-1}$ from Figure 2 which suggests that oxygen atoms under such conditions are able to sample appreciable parts of the available surface to eventually find reaction partners. Conversely, the desorption energy $E_{\mathrm{des}}=1000 \mathrm{~K}$ is sufficiently high to prevent escape from the surface and leads to long residence times.

In conclusion, the analysis of experimental and simulation results shows that oxygen diffusion on ASW down to very low temperatures is possible and does not require quantum tunneling. This insight is of fundamental relevance for the formation of molecules including $\mathrm{CO}_{2}$, water (minor pathway) and other molecules (e.g. $\mathrm{OCOH}$ from $\mathrm{HCO}$ or $\mathrm{OCCHO}$ from $\mathrm{HCCO})^{3}$ in laboratory experiments and on grains under conditions characteristic of the interstellar medium. Extension of such considerations to surfaces including graphite and silicon may provide further insight into chemical processing of grains in later stages of their development.

\section{Acknowledgment}

This work was supported by the Swiss National Science Foundation through grants 200021117810, and the NCCR MUST. The authors are grateful to Prof. F. Dulieu for providing us with raw experimental data and Profs. F. Dulieu and A. G. G. M. Tielens for insightful discussions. 


\section{References}

(1) Tielens, A. G. G. M.; Hagen, W. Model Calculations of the Molecular Composition of Interstellar Grain Mantles. Astron. Astrophys. 1982, 114, 245-260.

(2) Ruffle, D.; Herbst, E. New models of interstellar gas-grain chemistry - III. Solid CO2. Mon. Not. R. Astron. Soc. 2001, 324, 1054-1062.

(3) Charnley, S. B.; Rodgers, S. D. In Astrochemistry: Recent Successes and Current Challenges; Lis, D. C., Blake, G. A., Herbst, E., Eds.; International Astronomical Union, 2006; pp 237-246.

(4) Minissale, M.; Congiu, E.; Baouche, S.; Chaabouni, H.; Moudens, A.; Dulieu, F.; Accolla, M.; Cazaux, S.; Manico, G.; Pirronello, V. Quantum Tunneling of Oxygen Atoms on Very Cold Surfaces. Phys. Rev. Lett. 2013, 111, 053201.

(5) Cuppen, H. M.; Ioppolo, S.; Romanzin, C.; Linnartz, H. Water formation at low temperatures by surface O-2 hydrogenation II: the reaction network. Phys. Chem. Chem. Phys. 2010, 12, 12077-12088.

(6) Cuppen, H. M.; Herbst, E. Simulation of the formation and morphology of ice mantles on interstellar grains. Astrophys. J. 2007, 668, 294-309.

(7) Minissale, M.; Congiu, E.; Dulieu, F. Direct measurement of desorption and diffusion energies of $\mathrm{O}$ and $\mathrm{N}$ atoms physisorbed on amorphous surfaces. Astron. Astrophys. 2016, 585, A146.

(8) Congiu, E.; Minissale, M.; Baouche, S.; Chaabouni, H.; Moudens, A.; Cazaux, S.; Manico, G.; Pirronello, V.; Dulieu, F. Efficient diffusive mechanisms of O atoms at very low temperatures on surfaces of astrophysical interest. Faraday Discuss. 2014, $168,151-166$.

(9) Zwanzig, R. Diffusion on a rough potential. Proc. Natl. Acad. Sci. 1988, 85, 2029. 
(10) Dausinger, F.; Schultz, H. Long-range migration of self-interstitial atoms in tungsten. Phys. Rev. Lett. 1975, 35, 1773-1775.

(11) Swinburne, T. D.; Ma, P.-W.; Dudarev, S. L. Low temperature diffusivity of selfinterstitial defects in tungsten. New J. Phys. 2017, 19, 073024.

(12) Brooks, B. R. et al. CHARMM: The biomolecular simulation program. J. Comp. Chem. 2009, 30, 1545-1614.

(13) Lee, M. W.; Meuwly, M. Diffusion of atomic oxygen relevant to water formation in amorphous interstellar ices. Faraday Discuss. 2014, 168, 205-222.

(14) Jorgensen, W. L.; Chandrasekhar, J.; Madura, J. D.; Impey, R. W.; Klein, M. L. Comparison of Simple Potential Functions for Simulating Liquid Water. J. Chem. Phys. 1983, 79, 926-935.

(15) Ryckaert, J.-P.; Ciccotti, G.; Berendsen, H. J. C. Numerical integration of the cartesian equations of motion of a system with constraints: molecular dynamics of n-alkanes. $J$. Comp. Phys. 1977, 23, 327-341.

(16) Frisch, M. J. et al. Gaussian 09 Revision D.01. 2009; Gaussian Inc. Wallingford CT 2009.

(17) Cranck, J.; Nicolson, P. A practical method for numerical evaluation of solutions of partial differential equations of the heat conduction type. Proc. Camb. Phil. Soc 1947, $43,50-67$.

(18) Verlet, L. Computer "Experiments" on Classical Fluids. I. Thermodynamical Properties of Lennard-Jones Molecules. Phys. Rev. 1967, 159, 98-103.

(19) Karssemeijer, L. J.; Cuppen, H. M. Diffusion-desorption ratio of adsorbed CO and CO2 on water ice (Research Note). Astron. Astrophys. 2014, 569. 